\title{
Hybrid hydrogel photonic barcodes for multiplex detection of tumor markers
}

Yueshuang $\mathrm{Xu}^{1}$, Xiaoping Zhang ${ }^{1}$, Chengxin Luan ${ }^{1}$, Huan Wang ${ }^{2}$, Baoan Chen ${ }^{1 *}$, Yuanjin Zhao ${ }^{1,2^{*}}$

${ }^{1}$ State Key Laboratory of Bioelectronics, Department of Hematology and Oncology, Zhongda Hospital, School of Medicine, Southeast University, Nanjing 210009, China.

${ }^{2}$ School of Biological Science and Medical Engineering, Southeast University, Nanjing 210096, China.

* Corresponding author. Southeast University, Nanjing 210096, China.

E-mail addresses: cba8888@hotmail.com; yjzhao@seu.edu.cn 


\begin{abstract}
:
Barcodes-based suspension array have for demonstrated values in multiplex assay of tumor markers. Photonic barcodes which are encoded by their characteristic reflection peaks are the important supports for suspension array due to their stable code, low fluorescent background and high surface-volume ratio. Attempts to develop this technology tend to improve the function of the photonic barcodes. Here, we present a new type of hybrid hydrogel photonic barcodes for efficient multiplex assays. This photonic barcodes are hybrid inverse opal hydrogel composed of poly(ethylene glycol) diacrylate (PEG-DA) and agarose. The polymerized PEG-DA hydrogel could guarantee the stabilities of the inverse opal structure and its resultant code, while the agarose could offer active chemical groups for the probe immobilization and homogeneous water surrounding for the bioassay. In addition, the interconnected pores inverse opal structure could provide channels for biomolecules diffusing and reaction into the voids of barcodes. These features imparted the hybrid hydrogel photonic barcodes with limits of detection (LOD) of $0.78 \mathrm{ng} / \mathrm{mL}$ for carcinoembryonic antigen (CEA) and $0.21 \mathrm{ng} / \mathrm{mL}$ for $\alpha$-fetoprotein (AFP), respectively. It was also demonstrated that the proposed barcodes showed acceptable accuracy and detection reproducibility, and the results were in acceptable agreement with those from common clinic method for the detections of practical clinical samples. Thus, our technique provides a new platform for simultaneous multiplex immunoassay.
\end{abstract}

Keywords: tumor marker; barcode; hydrogel; multiplex assay; colloidal crystal. 


\section{Introduction}

Cancer has become one of the most devastating diseases worldwide due to its high mortality rates (Kanodra et al., 2015). People usually have terminal cancers when they are diagnosed because of lack of the incipient symptoms. Therefore, it's important to detect, treat and cure these terrible diseases earlier (Devi et al., 2015; Meng et al., 2015; Miller et al., 2014; Zheng et al., 2014). The tumor marker has significant roles in early screening of cancer, evaluating the severity of disease and monitoring the response of cancer therapy. However, because of its limited specificity, a single tumor marker is usually not sufficient to diagnose cancer. In this point, multiplex assay of tumor markers can avoid this dilemma to improve the diagnostic accuracy (Li et al., 2014b; Li et al., 2015; Liu et al., 2013; Zhao et al., 2009). To achieve the multiplexing, clinical laboratories still employ a parallel single-analyte assay by detecting one tumor marker at a time and then combining these results together. This method usually needs more sample consumption, longer assay time and higher cost.

Up to now, various approaches have been devised to realize simultaneous multiplex analysis (Freeman et al., 2013; Lee et al., 2014; Ye et al., 2014; Zou et al., 2015). A successful example of these approaches is the biochips, in which probes are bound on the flat substrate and encoded by their two-dimensional positions. In order to avoid some drawbacks of the biochips, such as slow diffusion of molecules and low sample throughput, barcodes based suspension arrays, in which probes are immobilized on the surface of fluorescence or quantum dots encoded microparticles, have become an alternative approach for multiplex assay (Li et al., 2014a; Yang et al., 2014; Yu et al., 2009; Zhang et al., 2006; Zhao et al., 2011). These barcodes have several advantages, including higher flexibility for new analytes and faster reaction kinetics in solution. However, the fluorescent 
or quantum dots barcodes are still with several disadvantages, including the photobleaching during storage and the potential interference of encoding fluorescence with analyte-detection fluorescence.

Recently, photonic crystal $(\mathrm{PhC})$ barcodes have been suggested as a new type of microcarriers for suspension array (Shang et al., 2015; Shen et al., 2011; Shi et al., 2013; Song et al., 2015; Wang et al., 2011; Zhao et al., 2014). As their encoded elements are the characteristic refection peaks originated from their periodical structure-induced photonic band gap (PBG), the $\mathrm{PhC}$ barcodes are extremely stable, and the controversial fluorescent signal is not aroused (Kanai et al., 2010; Lee et al., 2015; Sim et al., 2014, 2015; Yang et al., 2008; Zhao etal., 2015). These properties make them suitable for multiplex detection. However, most of the $\mathrm{PhC}$ barcodes are composed of silica nanoparticles or inert hydrogel, both of which are with dull surface chemistry and can only provide limited surfaces for the probe immobilization and target detection. These issues have restricted many practical applications of the $\mathrm{PhC}$ barcodes. Thus, the creation of novel $\mathrm{PhC}$ barcodes with active composed materials and functional structures are still anticipated.

In this paper, we proposed a new type of hybrid hydrogel $\mathrm{PhC}$ barcodes with these desired features (Figure 1). The hybrid hydrogel could be constructed by cross-linking distinct classes of polymer monomers, such as synthetic and biological macromolecules, through covalent or non-covalent method. Due to the elaborate combination of the different monomers, these hybrid hydrogels were usually imparted with distinct functions. Here, we employed a poly(ethylene glycol) diacrylate (PEG-DA) and agarose hydrogel to construct inverse opal PhC barcodes. The polymerized PEG-DA hydrogel could guarantee the stabilities of the inverse opal structure and its resultant structural color code, which impart the barcodes with distinctive encoded information for multiplex assays. The agarose could offer active chemical groups for the probe immobilization and 
homogeneous water surrounding for the bioassay (Jokerst et al., 2011). In addition, the interconnected pores inverse opal structure could provide channels for biomolecules diffusing and reaction into the voids of the barcodes. It was demonstrated that the $\mathrm{PhC}$ barcodes showed high accuracy, detection reproducibility, and acceptable agreement with common clinic method in the multiplex assay of tumor markers carcinoembryonic antigen (CEA) and $\alpha$-fetoprotein (AFP) for practical clinical samples. Thus, this new $\mathrm{PhC}$ barcodes would find many applications in analytical and clinical areas.

\section{Experimental section}

\subsection{Materials}

Human AFP, CEA, mouse monoclonal anti-human AFP antibody, anti-human CEA antibody were purchased from Shenzhen Constant Medical Engineering Company, China. Cy3 labeled rabbit polyclonal anti-human AFP antibody, anti-human CEA antibody were purchased from Micro Biological Technology Company, Shanghai, China. Bovine serum albumin (BSA) was obtained from Sigma-Aldrich, Shanghai, China. Clinical serum samples were obtained from Zhongda Hospital, China. Phosphate buffer saline (PBS, 0.05M, PH 7.4) and PBS containing 0.05\% Tween-20 (PBST) were self-prepared. Epoxy chloropropane $(\mathrm{ECH})$ was purchased from Aladdin Industrial Corporation. $2 \mathrm{M} \mathrm{NaOH}$ was used for activating agarose with ECH. Agarose was purchased from Biosharp Company, America. Poly (ethylene glycol) diacrylate (PEG-DA) with molecular weights of 700 and 2-hydroxy-2-methylpropiophenone (HMPP) photoinitiator were purchased from Sigma-Aldrich, Shanghai, China. All buffers were self-prepared using water purified in a Milli-Q system (Millipore, Bedford, USA). 


\subsection{Instruments}

The microfluidic device used for generating silica colloidal crystal beads was home-made. All reactions were finished in flat-bottom tubes and a constant temperature shaker (Thermomixer comfort 5355, Eppendorf, Germany). The microstructures of silica colloidal crystal beads and hybrid hydrogel photonic barcodes were characterized according to a scanning electron microscope (SEM, S-300N, Hitachi, Japan). Photographs of the two kinds of beads were taken by an optical microscope (BX51, Olympus, Japan) equipped with a CCD camera (MP5.0, Media Cybernetics Evolution). The reflectance spectra of the barcodes were recorded by the same microscope equipped with a fiber optic spectrometer (HR2000, Ocean Optics, USA). The fluorescence intensity was detected by a fluorescence microscope (BX53, Olympus, Japan).

\subsection{Preparation of the hybrid hydrogel PhC barcodes}

Silica colloidal crystal beads used as the template to fabricate this kind of $\mathrm{PhC}$ barcodes were fabricated using the microfluidic devices as mentioned before. The agarose was dissolved in purified water with PEG-DA and HMPP in $80{ }^{\circ} \mathrm{C}$ water bath. Then the dried silica colloidal crystal beads with different colors were immersed in heated agarose solution prepared before for $1 \mathrm{~h}$. The liquid mixed solution could fill the gaps of silica colloidal crystal beads fully. Next, the mixture of beads and pre-gel solution was exposed to UV light for polymerizing the pre-gel solution. After the agarose was cooled, the hybrid beads of different colors could be extracted by stripping the pre-gel on the surface of the beads. Finally, the PhC barcodes we want could be obtained by removing the silica colloidal crystal beads with $4 \%$ hydrofluoric acid.

\subsection{Probes immobilization.}

Firstly, the PhC barcodes were treated with solution containing $10 \% \mathrm{ECH}$ and $1 \% \mathrm{NaOH}$ for 3 
h at $37{ }^{\circ} \mathrm{C}$ in the constant shaker. This reaction could offer epoxy group of ECH which was conjugated to the hydrogel beads via a single replacement reaction of chlorine atom. Then two kinds of barcodes with different reflection peak positions of 576 and $670 \mathrm{~nm}$ were separately coated with mouse anti-human AFP antibody or CEA antibody after washing with water overnight at $25{ }^{\circ} \mathrm{C}$. Finally, the different functionalized PhC barcodes with own probes were prepared successfully after washing with buffer solution.

\subsection{Detection of tumor markers}

For single detection, firstly, the prepared $\mathrm{PhC}$ barcodes should be treated with BSA for $2 \mathrm{~h}$. Then, functionalized $\mathrm{PhC}$ barcodes were incubated with different concentrations of tumor marker (diluted in PBS) in the flat-bottom tubes for 30min. Finally, Cy3 labeled rabbit anti-tumor marker antibody $(0.1 \mathrm{mg} / \mathrm{mL})$ was added into the flat-bottom tubes and incubated for $30 \mathrm{~min}$. During the whole detection process, the tubes were shaken at $25{ }^{\circ} \mathrm{C}$ in the constant shaker, and this three steps should be followed by washing away unbound molecules thoroughly with buffer solution. The number of beads at any concentration was 5 and the volume of the tumor marker and anti-tumor marker antibody added into the test tubes was $1 \mu \mathrm{L}$ per bead.

For multiplexed detection, two kinds of PhC barcodes with reflection peak position in 576 and $670 \mathrm{~nm}$, coated with mouse anti-human AFP antibody and anti-human CEA antibody, were put into one flat-bottom tubes for testing. The sample was added and incubated for 30min.After being washed with PBS buffer, the PhC barcodes were added into the mixed solution of two Cy3 labeled rabbit anti-tumor marker antibodies followed by washing the barcodes thoroughly with PBS buffer again. Both steps were carried out in the dark with constant shaking at $25^{\circ} \mathrm{C}$.

For the detection by ECLIA, the probes were firstly immobilized on a substrate. Then the blood 
samples were added for the targets capture by the specific antigen-antibody reaction. After the reaction and buffer washing, electrochemical luminescence agent $\mathrm{Ru}(\mathrm{bpy}) 3$ labelled second antibody was added for incubating. Finally, the target concentration was read out by using chemiluminescent analytical system after adding voltage to the electrode.

\section{Results and discussion}

\subsection{Design of the hybrid hydrogel PhC barcodes.}

As a typical experiment, the hybrid inverse opal hydrogel microcarriers were fabricated by replicating silica colloidal crystal beads. In this process, the silica colloidal crystal bead templates were immersed in the pre-gel solution firstly. Then the pre-gel could go through the voids between the nanoparticles of the templates by the capillary force. After the pre-gel solution filled the void fully, the pre-gel solution in and out of the templates was polymerized by exposing to UV light or changing the temperature. Finally, the PhC barcodes were obtained by removing the pre-gel on the surface of the templates and etching the templates by hydrofluoric acid.

To improve the function of the photonic barcodes, we employed the mixture of poly(ethylene glycol) diacrylate (PEG-DA) and agarose as the scaffold material for the PhC barcodes (schematic in Figure 1a). PEG-DA has some biological characteristics, such as non-toxicity, mechanical strength and resistance of protein. Thus, it can guarantee the stability of the inverse opal structure and eliminate the nonspecific binding of proteins to meet the demand of specific biomolecular screening. Agarose was incorporated into the scaffold due to its perfect biocompatibility, which can not only offer a great deal of hydroxyl groups for the probes immobilization, but also provide homogeneous 
water surrounding for improving the bio-reaction efficiency of the probes and their corresponding targets. It was worth to mention that the interconnected pores inverse opal structure of the $\mathrm{PhC}$ barcodes could provide channels for biomolecules diffusing and reaction into the voids of whole particles, which made the barcodes more advantages than traditional hydrogel microcarriers.

\subsection{Fabrication of the hybrid hydrogel PhC barcodes.}

During the $\mathrm{PhC}$ barcodes fabrication, both of the concentration of the PEG-DA and agarose could influence the quality of the particles. To optimize the concentration of the PEG-DA, five kinds of $\mathrm{PhC}$ barcodes with different concentration of PEG-DA were fabricated by the template replication of the same silica colloidal crystal beads with reflection peak position in 670nm, as shown in Figure S1. It was found that lower concentration of PEG-DA could offer bigger pores to make biomolecules diffusing easier. However, when the concentration of PEG-DA was lower than $20 \%$, the PhC barcodes showed poor mechanical properties, strong adhesion to the centrifuge tube, which is not benefit for the next experiment processes of biomolecule assay and unobvious structural colors (Figure S1d-e). Moreover, the PhC barcodes with $10 \%$ or 5\% PEG-DA even didn't have good reflection peak, which was not benefit for code in multiplex detection (Figure S1f). In comparable, the PhC barcodes composed of 20\% PEG-DA exhibited strong mechanical properties, inferior adhesion and obvious structural colors (Figure S1c). Therefore, this concentration of the PEG-DA was used to fabricate all the $\mathrm{PhC}$ barcodes for the following experiment.

For the concentration of the agarose, the lower one couldn't offer the good mechanical properties, while the upper one could provide more hydroxyl groups for capturing biological molecules. However, the hydrogel $\mathrm{PhC}$ barcodes would lose their elasticity and become frangible when they were composed of a high concentration of the agarose. With a series of optimized test, it was found 
that $8 \%$ agarose was the moderate one concerning these two problems. Thus, we employed this concentration of agarose to produce all the hybrid hygrogel $\mathrm{PhC}$ barcodes for the biological study.

To confirm the microstructure of the fabricated particles, the surface of the silica colloidal crystal bead templates and the PhC barcodes were observed with a scanning electron microscope (SEM). It can be seen from Figure 2 that the nanoparticles on the surface of the silica colloidal crystal beads mainly formed a hexagonal alignment (Figure 2a), and the $\mathrm{PhC}$ barcodes replicated from the templates had a similar highly ordered three dimensional (3D) inverse opal structure and hexagonal symmetrical porous surface (Figure $\mathbf{2 b}$ ). This structure would provide a nanopatterned platform with larger surface area for highly efficient entrapping biomolecules.

\subsection{Encoded Reflection Spectra of the PhC barcodes.}

The encoded reflection peaks of the PhC barcodes mostly depend on the structural periodicity of the nanopores. Because of their ordered nanostructure, the hybrid hydrogel inverse opal $\mathrm{PhC}$ barcodes were imparted with a photonic band gap (PBG) property and show the corresponding structural color or characteristic reflection peak. Under normal incidence, the peak positions $\lambda$ of the $\mathrm{PhC}$ barcodes can be estimated by Bragg's equation:

$$
\lambda=1.633 d n_{\text {average }}
$$

where $d$ is the center-to-center distance between two neighboring nanopores, and $n_{\text {average }}$ is the average refractive index of the PhC barcodes. As the $n_{\text {average }}$ of our PCBs is a constant when the composed hydrogel was fixed, the peak value $\lambda$ mainly depends on the size of the nanopores. Therefore, by changing the diameters of the silica nanoparticles and their derived nanopores, a series of $\mathrm{PhC}$ barcodes with different peak wavelengths and colors could be obtained.

Figure 3 showed the reflection images and spectra of three kinds of silica colloidal crystal 
beads with reflection peak positions in about 670nm, 576nm and 465nm, respectively. These PhC barcodes were with the vivid structure colors of red, green and blue. It was found that after replicate these bead templates with the hybrid hydrogel of the agarose and PEG-DA, the peak wavelengths of the achieved hydrogel barcodes did not change obviously. Therefore, the PhC barcodes were still with their different reflection peak positions, and thus could be used as code elements for multiplex detection. In addition, there was no dye or other materials related with code, so we needn't worry about chemical instability and the fluorescence background.

\subsection{Optimization of reaction conditions.}

Before coating with probes, the barcodes should be activated in the mixture of Epoxy chloropropane $(\mathrm{ECH})$ and $\mathrm{NaOH}$. During this process, there were three aspects should be taken into consideration to improve our study, including the concentration of $\mathrm{ECH}$, the concentration of $\mathrm{NaOH}$ used in the active reaction, and the incubation time in this step. Figure $\mathbf{S 2}$ showed the relationships of fluorescence intensities with these three factors. Here we used human IgG as the target protein, and two different goat polyclonal anti-human IgG antibodies as the capture antibody and reporter antibody. Figure S2a showed that the fluorescence intensity reached the peak value when the concentration of ECH was $10 \%$, while concentration of $\mathrm{NaOH}$ here was fixed at $1 \mathrm{M}$ and the incubation time was fixed at $3 \mathrm{~h}$. Figure S2b showed that the fluorescence intensity reached the maximum value when the concentration of $\mathrm{NaOH}$ was $1 \mathrm{M}$, while the concentration of ECH was fixed at $10 \%$ and the incubation time was fixed at $3 \mathrm{~h}$. Figure S2c showed that with incubation time increasing, the fluorescence intensities increased quickly and reached maximum value at $3 \mathrm{~h}$ when the concentration of $\mathrm{ECH}$ and $\mathrm{NaOH}$ were fixed at $10 \%$ and $1 \mathrm{M}$. In order to confirm this optimal reaction condition, orthogonal design and experiments have been implemented. We chose three 
levels for every factor: the optimal one mentioned before, larger one and smaller one than that. The results were shown in Table S1. It indicated that the fluorescence intensity was higher when the concentration of $\mathrm{ECH}$ was more than $10 \%$, the concentration of $\mathrm{NaOH}$ was more than $1 \mathrm{M}$ and the reaction time was more than $3 \mathrm{~h}$. Thus, in the following study, we activated the barcodes in the mixture of $10 \% \mathrm{ECH}$ and $1 \mathrm{M} \mathrm{NaOH}$ for $3 \mathrm{~h}$.

\subsection{Tumor marker detection.}

Cancer has become one of the most devastating diseases worldwide with the high mortality rates. Detection of tumor markers is convenient and has little trauma, just takes a little blood from you. And it is not so expensive compared with other exams, so it is more acceptable by people. Thus, we here chose AFP and CEA as the typical multiplex combination of tumor markers for the detection. In clinic practice, the high level of AFP and CEA were found in these cancers, including liver cancer, gastrointestinal cancer or lung cancer. They don't have the specific for a single tumor. Also, many patients usually get metastatic cancers which involve several system cancers in body. Thus, the multiplex detection of AFP and CEA is important for diagnosis, metastasis and prognosis.

Under optimal conditions, we detected routine samples of these two tumor markers with different concentrations ranging from 0.1 to $10000 \mathrm{ng} / \mathrm{mL}$. Figure 4 showed the dose-response curves for single detection of CEA and AFP. It was found that although the curves were not linear, but the fluorescence intensity became higher as the concentration of analyte was increased from $1 \mathrm{ng} / \mathrm{mL}$ to $1000 \mathrm{ng} / \mathrm{mL}$. The limits of detection (LOD) of the hybrid hydrogel photonic barcodes were $0.78 \mathrm{ng} / \mathrm{mL}$ for CEA and $0.21 \mathrm{ng} / \mathrm{mL}$ for AFP, while the cut-off values of AFP and CEA of clinical diagnosis in Zhongda Hospital are $8 \mathrm{ng} / \mathrm{mL}$ and $5 \mathrm{ng} / \mathrm{mL}$, respectively. Thus, the detection ranges of our method here was enough for practical application. 
Cross-reactivity is important for reliability of multiplex immunoassay. In this study, two tumor markers, AFP and CEA, should be mixed and detected in one flat-bottom test tube, and the cross reactivity would influence the reliability of the multiplex immunoassay. The cross reactivity in this research was evaluated with the fluorescence signals which were detected when the concentration of the specific analyte was constant while the concentration of another one was increased, respectively. Figure 5 showed the fluorescence intensities for specific tumor markers at a concentration of $100 \mathrm{ng} / \mathrm{mL}$ while the concentration of another increased to $500 \mathrm{ng} / \mathrm{mL}$. It could be seen that the maximum changes of the fluorescence intensities for each didn't go beyond 5\%, respectively. The results recommended that the cross-reactivity between AFP and CEA could be neglected and we could realize the multiplex immunoassay.

\subsection{Clinical detection of the PhC barcodes.}

To check the application potential and the reliability of the hybrid hydrogel $\mathrm{PhC}$ barcodes for multiplex assay in clinic, we compared this method with the common method in clinic laboratory called electrochemiluminescence immunoassay (ECLIA). We examined almost 40 serum samples which were centrifuged from blood cells from 40 patients drawn by using the standard venipuncture technique in Zhongda Hospital, China. Among these patients, almost 20 suffered digestive system cancers and the other 20 suffered lung cancer. Figure 6 showed the results. The regression equations (linear) for these data were as follows (x axis, ECLIA; y axis, PhC barcodes):

$\mathrm{y}=-47.8798+1.2070 \times(\mathrm{r} 2=0.9494)$ for AFP for digestive cancer

$\mathrm{y}=-2.7102+1.0025 \times(\mathrm{r} 2=0.9666)$ for CEA for digestive cancer

$\mathrm{y}=0.1962+1.0364 \times(\mathrm{r} 2=0.9573)$ for AFP for lung cancer

$y=-1.3935+0.938 \times(r 2=0.9699)$ for CEA for lung cancer 
It can be found that the relative standard deviation (RSD) of the intra- and inter-assay were about $0.1 \%$ and $8.83 \%$. The accuracy for the four figures in Figure 6 were $7.05 \%, 8.36 \%, 14.77 \%$ and $10.36 \%$, respectively. The precision were $4.81 \%, 5.89 \%, 18.28 \%$ and $6.31 \%$, respectively. Although our biosensor didn't show the same good accuracy, precision, and reproducibility as ECLIA, it can realize multiplex detection simultaneously in a tube, which was also with satisfied detection sensitivity for practical application.

These data indicated that there was no significant difference for the detection results between the two methods, which meant our method had good reliability and application potential. It also could be seen that most of the patients who suffered digestive cancers had high concentration both of AFP and CEA, but patients who had lung cancer only had high concentration of CEA. In addition, this method could finish the multiplex immunoassay of the two tumor markers in one test tube and needed fewer samples than ECLIA.

\section{Conclusion}

In conclusion, a novel PEG-DA and agarose hybrid hydrogel photonic barcodes with improved features has been developed for multiplex detection of tumor markers. The polymerized PEG-DA hydrogel guaranteed the stabilities of the inverse opal structure and its resultant code, the agarose offered active chemical groups for the probe immobilization and homogeneous water surrounding for the bioassay, while the inverse opal structure provided more surface area and interconnected pore channels for biomolecules diffusing and reaction into the voids of barcodes. The reaction and detection conditions have been investigated and optimized. The dose-response curves of the tumor markers (AFP and CEA) with sufficient detection ranges were obtained based on the photonic 
barcodes. It was also demonstrated that these barcodes were with acceptable accuracy and detection reproducibility in the multiplex assay of the practical clinical samples. These features of the hybrid hydrogel $\mathrm{PhC}$ barcodes make them excellent suspension array, and thus they are anticipated to open new horizons in medical diagnostics.

\section{Acknowledgment}

This work was supported by the National Science Foundation of China (Grant Nos. 81370673, 21473029 and 51522302), Key Medical Projects of Jiangsu Province (Grant No. H201336), the National Science Foundation of Jiangsu (Grant No. BK20140028), and the Scientific Research Foundation of Southeast University.

\section{References}

Devi, R.V., Doble, M., Verma, R.S., 2015. Biosens. Bioelectron. 68, 688-698.

Freeman, R., Girsh, J., Willner, I., 2013. Acs Appl. Mater. Inter. 5, 2815-2834.

Jokerst, J.V., Chou, J., Camp, J.P., Wong, J., Lennart, A., Pollard, A.A., Floriano, P.N., Christodoulides, N., Simmons, G.W., Zhou, Y.J., Ali, M.F., McDevitt, J.T., 2011. Small 7, 613-624.

Kanai, T., Lee, D., Shum, H.C., Weitz, D.A., 2010. Small 6, 807-810.

Kanodra, N.M., Silvestri, G.A., Tanner, N.T., 2015. Cancer 121, 1347-1356.

Lee, J., Bisso, P.W., Srinivas, R.L., Kim, J.J., Swiston, A.J., Doyle, P.S., 2014. Nat. Mater. 13, 524-529.

Lee, S.S., Kim, S.K., Won, J.C., Kim, Y.H., Kim, S.H., 2015. Angew. Chem. Int. Edit. 54, 15266-15270.

Li, J., Qi, H.M., Wang, H., Yang, Z.J., Zhu, P.Z., Diao, G.W., 2014a. Microchim. Acta 181, 
1109-1115.

Li, J., Wang, H., Dong, S.J., Zhu, P.Z., Diao, G.W., Yang, Z.J., 2014b. Chem. Commun. 50, 14589-14592.

Liu, N., Liang, W.F., Ma, X.H., Li, X.L., Ning, B.A., Cheng, C.N., Ou, G.R., Wang, B.G., Zhang, J., Gao, Z.X., 2013. Biosens. Bioelectron. 47, 92-98.

Li, P., Zhang, B., Cui, T.H., 2015. Biosens. Bioelectron. 72, 168-174.

Meng, J.X., Zhang, P.C., Zhang, F.L., Liu, H.L., Fan, J.B., Liu, X.L., Yang, G., Jiang, L., Wang, S.T., 2015. Acs Nano 9, 9284-9291.

Miller, J.W., Hanson, V., Johnson, G.D., Royalty, J.E., Richardson, L.C., 2014. Cancer 120, 2549-2556.

Shang, L.R., Fu, F.F., Cheng, Y., Wang, H., Liu, Y.X., Zhao, Y.J., Gu, Z.Z., 2015. J. Am. Chem. Soc. 137, 15533-15539.

Shen, W.Z., Li, M.Z., Xu, L., Wang, S.T., Jiang, L., Song, Y.L., Zhu, D.B., 2011. Biosens. Bioelectron. 26, 2165-2170.

Shi, X.D., Li, M.Z., Ye, C.Q., Shen, W.Z., Wen, Y.Q., Chen, L.F., Yang, Q., Shi, L., Jiang, L., Song, Y.L., 2013. Laser Photonics Rev. 7, L39-L43.

Sim, J.Y., Choi, J.H., Lim, J.M., Cho, S., Kim, S.H., Yang, S.M., 2014. Small 10, 3979-3985.

Sim, J.Y., Lee, G.H., Kim, S.H., 2015. Small 11, 4938-4945.

Song, Y., Chan, Y.K., Ma, Q.M., Liu, Z., Shum, H.C., 2015. Acs Appl. Mater. Inter. 7, $13925-13933$.

Wang, J.X., Zhang, Y.Z., Wang, S.T., Song, Y.L., Jiang, L., 2011. Accounts Chem. Res. 44 $405-415$

Yang, Z.J., Luo, S.F., Li, J., Shen, J., Yu, S.H., Hu, X.Y., Dionysiou, D.D., 2014 Anal. Chim. Acta 839, 67-73.

Yang, Z.J., Xie, Z.Y., Liu, H., Yan, F., Ju, H.X., 2008. Adv. Funct. Mater. 18, 3991-3998.

Ye, B.F., Ding, H.B., Cheng, Y., Gu, H.C., Zhao, Y.J., Xie, Z.Y., Gu, Z.Z., 2014. Adv. Mater. 26, $3270-+$

Yu, H.K., Mao, Z.W., Wang, D.Y., 2009. J. Am. Chem. Soc. 131, 6366-+.

Zhang, H., Edwards, E.W., Wang, D.Y., Mohwald, H., 2006. Phys. Chem. Chem. Phys. 8, 
3288-3299.

Zhao, Y.J., Cheng, Y., Shang, L.R., Wang, J., Xie, Z.Y., Gu, Z.Z., 2015. Small 11, 151-174.

Zhao, Y.J., Shang, L.R., Cheng, Y., Gu, Z.Z., 2014. Accounts Chem. Res. 47, 3632-3642.

Zhao, Y.J., Shum, H.C., Chen, H.S., Adams, L.L.A., Gu, Z.Z., Weitz, D.A., 2011. J. Am. Chem. Soc. 133, 8790-8793.

Zhao, Y.J., Zhao, X.W., Pei, X.P., Hua, J., Zhao, W.J., Chen, B.A., Gu, Z.Z., 2009. Anal. Chim. Acta 633, 103-108.

Zheng, F.Y., Cheng, Y., Wang, J., Lu, J., Zhang, B., Zhao, Y.J., Gu, Z.Z., 2014. Adv. Mater. 26, 7333-7338.

Zou, F.M., Zhou, H.J., T, T.V., Kim, J., Koh, K., Lee, J., 2015. Acs Appl. Mater. Inter. 7, 12168-12175 


\section{Figure captions}

Figure 1. (a) Schematic diagram of the structure of the hybrid hydrogel $\mathrm{PhC}$ barcodes and the procedure of single marker detection. The hybrid hydrogel $\mathrm{PhC}$ barcodes are composed of agarose and PEG-DA. The probe antibodies are immobilized on the barcodes by hydroxyl groups of agarose.

(b) The procedure of multiplex detection based on the optical encoding property of the barcodes.

Figure 2. SEM images: (a) surface structure of the silica colloidal crystal beads, which indicated a commendable hexagonal close packing structure; (b) hydrogel $\mathrm{PhC}$ barcodes with an interconnected porous surface.

Figure 3. (a-b, d-e, g-h) Reflection images of three kinds of silica colloidal crystal beads and their corresponding hydrogel $\mathrm{PhC}$ barcodes, respectively: (a, d, g) silica colloidal crystal beads with reflection peak positions in about 670nm, 576nm and 465nm; (b, e, h) PhC barcodes. (c, f, i) Reflection spectra of the three kinds of the hybrid hydrogel PhC barcodes.

Figure 4. Calibration plots of fluorescence intensity vs. tumor marker concentrations: (a) AFP; (b) CEA. The number of replicates at any concentration was five. Error bars represent standard deviations.

Figure 5. The cross-reactivity among the two tumor markers. They were examined by comparing the fluorescence signals at a concentration $(100 \mathrm{ng} / \mathrm{mL})$ of specific analyte with increasing levels of another coexistent analyte, respectively. The number of replicates at any concentration was five. 
Error bars represent standard deviations.

Figure 6. Correlation between the proposed system and the standard ECLIA method for two tumor marker measurements of 40 clinical samples, of which half suffered digestive cancers and another half suffered lung cancer: (a, b) digestive cancers; (c, d) lung cancer. The number of replicates of any clinical sample was five. Error bars represent standard deviations. The error ranges of values measured using ECLIA was about 3\%. 
(a) Capture Antibody Target Protein $\quad \begin{aligned} & \text { Reporter Antibody } \\ & \text { With Fluorescence }\end{aligned}$

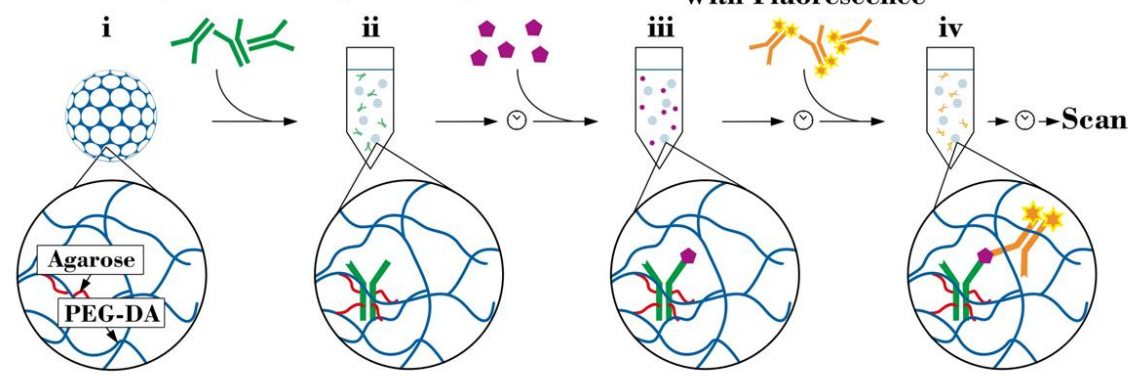

(b)
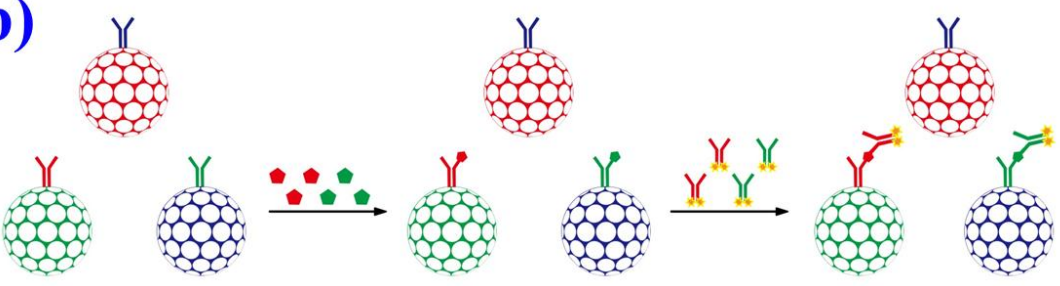

- human CEA

human AFP

Y anti-human CEA Ab Y anti-human AFP Ab Y anti-human NSE Ab

Y) Cy3 labeled anti-human CEA Ab II Cy3 labeled anti-human AFP Ab

Figure 1

Xu et al. 


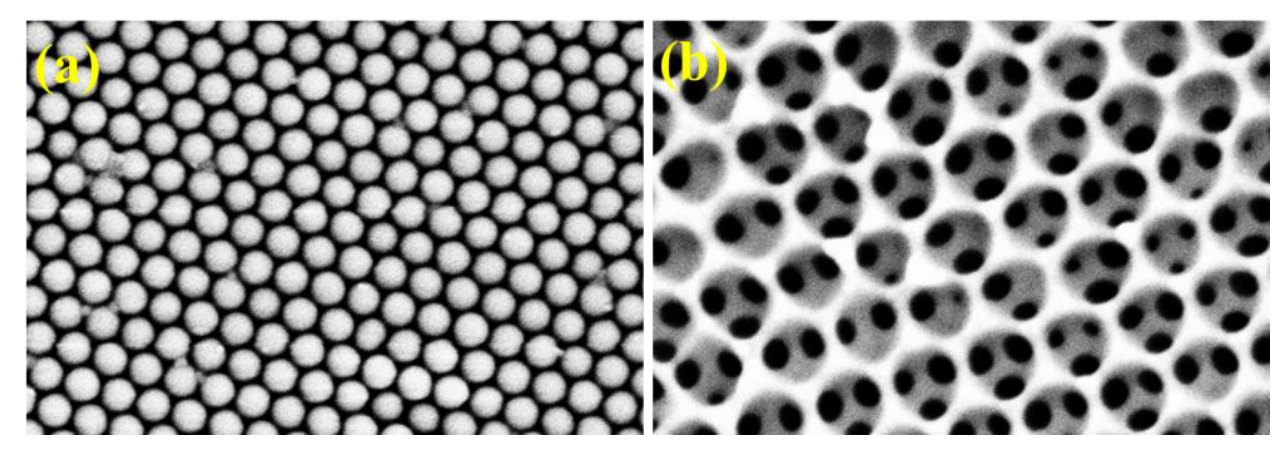

Figure 2

Xu et al. 

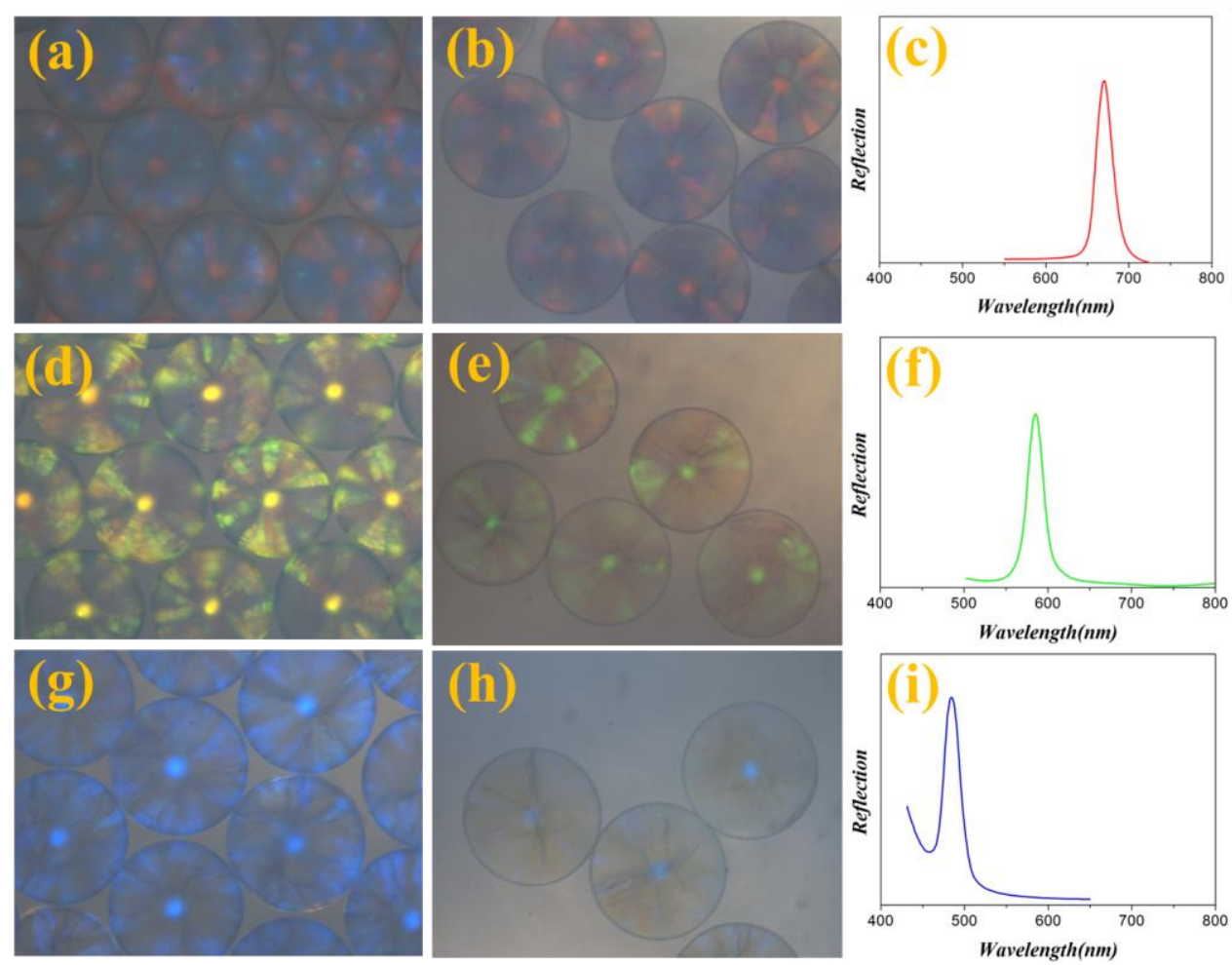

Figure 3

Xu et al. 

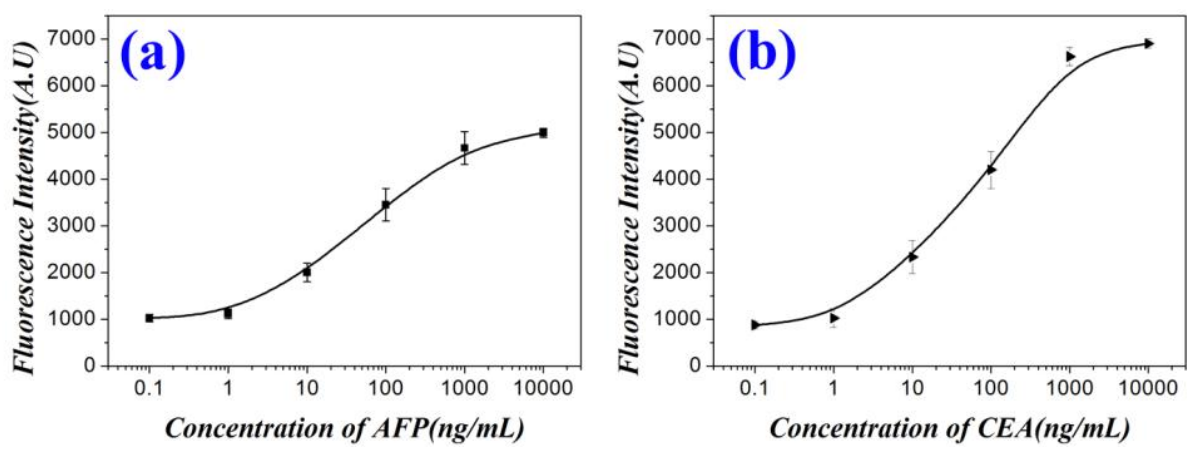

Figure 4

Xu et al. 


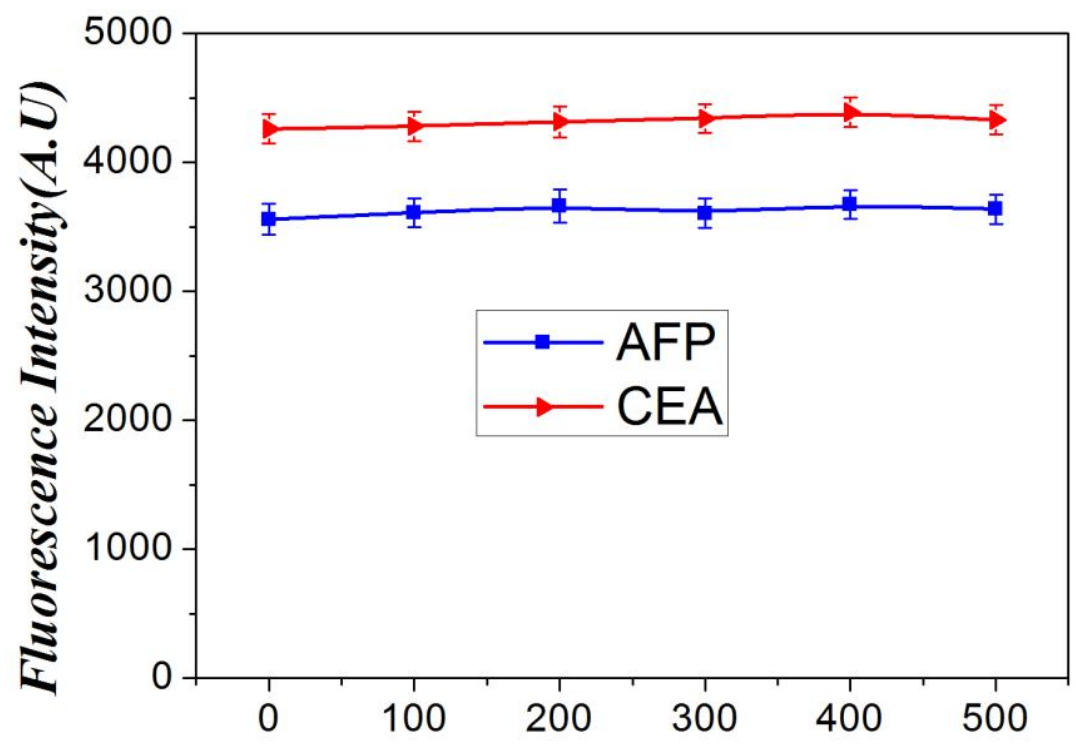

Concentration of coexistent analytes $(\mathrm{ng} / \mathrm{mL})$

Figure 5

Xu et al. 

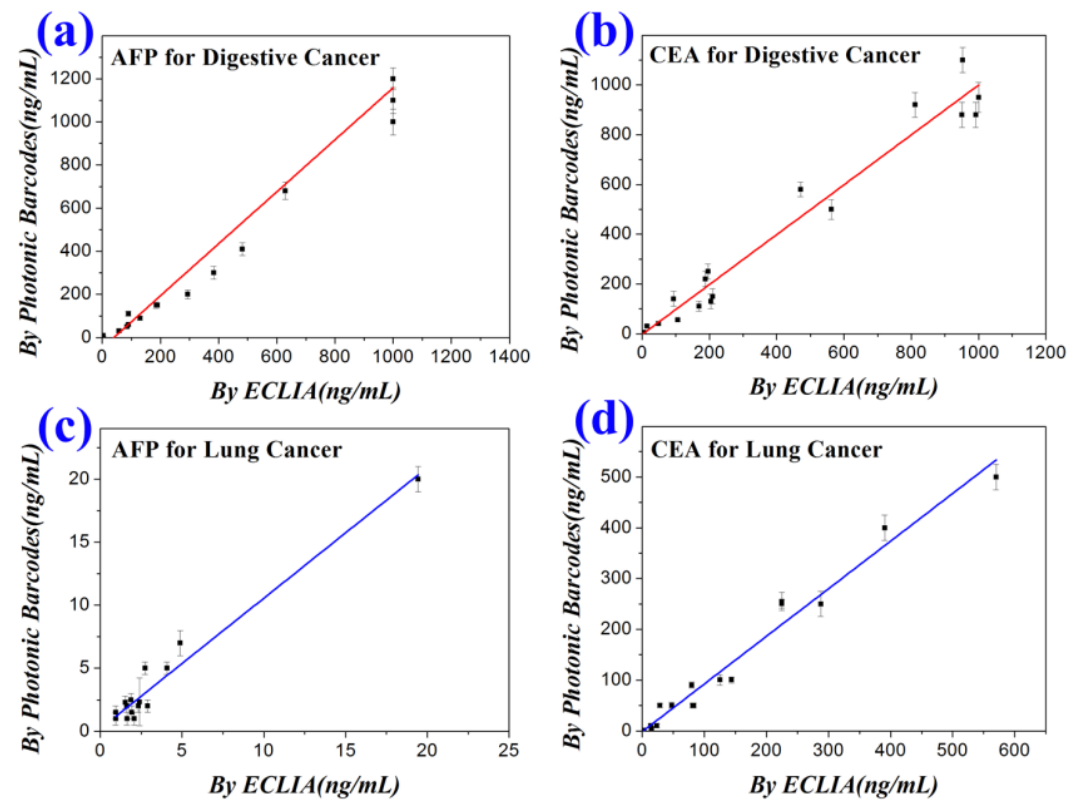

Figure 6

Xu et al. 